\title{
Clinical and electrophysiological aspects of tics in children
}

\author{
G.I. Safiullina* and A.A. Safiullina \\ Kazan State Medical Academy, Neurology, reflexotherapy and Osteopathy Sub-faculty, Kazan, Russia \\ *Corresponding author. E-mail: g.i.safiullina@mail.ru
}

BACKGROUND. Tics are diverse in nature inappropriate movements or vocalizations. They significantly degrade patients' quality of life, lead to social difficulties, and disturbance of learning especially during exacerbations. The prevalence of tics among children ranges from $4 \%$ to $24 \%$, thus emphasizing the relevance of the problem.

OBJECTIVE: To study clinical and electrophysiological features of tics in children with development of new treatment methods.

METHODS: We conducted a comprehensive clinical and electrophysiological examination of 50 patients with tics, aged 5 to 15 years. The control group consisted of 20 healthy children. The research included a thorough study of the history, neurological examination, manual testing of skeletal muscles, psychological testing. Electrophysiological examination included a review of the functional state of corticospinal tract (CST) by the method of magnetic stimulation (MS), study of polysynaptic reflex excitability (PRE) according to a late component of the blink reflex (BR). Statistical analysis included parametric and nonparametric methods of data processing.

RESULTS. All children of the study group showed signs of minimal brain dysfunction (MBD), they had complicated antenatal and postnatal history (trauma, disease, occurring with intoxication). There was a trend towards the increase of MBD signs with worsening of tics. Manual diagnosis in patients identified functional blockade at different levels of the vertebral column, sacroiliac joints, we identified latent myofascial trigger points (MFTP) mainly in the cervical-collar zone, in the area of the paravertebral muscles, periosteal triggers in the area of the sacroiliac joints.

The research allowed determining decrease in propagation velocity of excitation (PVE) throughout $\mathrm{CST}$ in patients with tics. Correlation analysis revealed a negative correlation between the severity of tics and PVE $(r=-0.38 ; p<0.001)$.

When studying polysynaptic reflex excitability (PRE) a significant predominance of hyper-excited types of blink reflex (BR) (90\% of cases) was revealed. However, in $10 \%$ of patients there was a moderate decrease in propagation velocity of excitation (PVE), which allowed us to identify two subgroups of patients with tics: I - low and moderate type of reflex responses; and II - high type of reflex responses. Collation of data of MS and BR revealed a significant decrease of PVE in patients of the subgroup I, which probably reflects a deeper disturbance of the neuro-motor apparatus. The presence of numerous myofascial trigger points (MFTP) in patients of the subgroup I with moderately low polysynaptic reflex excitability $(p<0.05)$ was characteristic. 
CONCLUSIONS: The data show extraordinary sensitivity of neuromuscular system of children to various physiological and pathological stimuli, occurring in the body in the ontogenesis or diseases, and multifactorial origin of the pain syndrome in tics.

The results suggest that one of the main mechanisms of development of pathological process is dysfunction of descending inhibitory control. However, further clarification of the type of polysynaptic reflex excitability in a certain patient is needed, that will allow to develop individualized approach to the choice of therapeutic interventions.

Keywords: Tics, children, excitability, reflex, blink reflex

\section{Conflict of interest statement: None.}

